\title{
Xanthogranulomatous Pyelonephritis Masque raging as Renal Cell Carcinoma - a Case Report
}

\author{
Authors \\ Dr Shankar Gunadal, Dr Suresh Bhat, Dr Fredrick Paul, Dr Suyog Sheety, \\ Dr Arun P.R. \\ GMC Kottayam
}

\begin{abstract}
A 47-year-old diabetic patient presented with left abdominal pain of 4 months duration. Evaluation of the patient revealed complex cystic mass in the left kidney, suggestive of cystic renal cell carcinoma. Radical nephrectomy was performed. Histopathology revealed xanthogranulomatous pyelonephritis.

Keywords: Chronic kidney infection, renal cell carcinoma, xanthogranulomatous pyelonephritis.
\end{abstract}

\section{Introduction}

Xanthogranulomatous pyelonephritis (XGP) is an uncommon, destructive and serious subtype of chronic pyelonephritis. Its clinical presentation is variable and hence it is called "a greater mimic". It is present in $<1 \%$ of RCC patients. XGP usually involves the entire kidney occasionally focal XGP has been reported. Focal renal involvement may be found without stones, obstruction and documented infection ${ }^{1}$. As there are no specific clinical and radiological findings, it is often misdiagnosed as renal cell carcinoma (RCC).

XGP shares many characteristics with true renal neoplasm in terms of its radiographic appearance and its ability to involve adjacent structures or organs. There are no specific radiological signs suggestive of XGP. Not infrequently radical surgery is done in these patients.

\section{Case Report}

A 47-years diabetic gentleman presented with left abdominal pain of 4 months duration. Physical examination did not reveal any findings. Routine investigations showed $\mathrm{Hb} \%$ - 10.2 gm\%, total count -10000cells/cumm, serum creatinine - 1.5 $\mathrm{mg} / \mathrm{dl}, \mathrm{LDH}-512 \mathrm{IU} / \mathrm{L}$. Urine culture did not grow any organism. Ultrasonography (US) abdomen showed a heterogeneous mass of $9.6 \mathrm{x}$ $6.6 \times 8.1 \mathrm{cms}$ with predominantly cystic lesion involving the lower half of left kidney. Contrast enhanced computed tomography (CECT) showed cystic lesion with septations and irregular wall in the lower pole of left kidney measuring 9 x $8.7 \times$ $7.8 \mathrm{cms}$, cyst adherent to adjacent psoas and paraspinal muscles [Fig - I]. Radical nephrectomy was performed with a diagnosis of Bosniak category IV cyst. Cut specimen extruded greenish yellow thick fluid. Microscopy showed cyst wall composed of fibrocollagenous tissue with dense inflammatory infiltrates predominantly plasma cells, eosinophills, lymphocytes, multinucleated gaint cells and foamy histiocytes. Adjacent kidney showed evidence of chronic pyelonephritis [Fig- 
II]. These findings confirmed the diagnosis of xanthogranulomatous pyelonephritis.

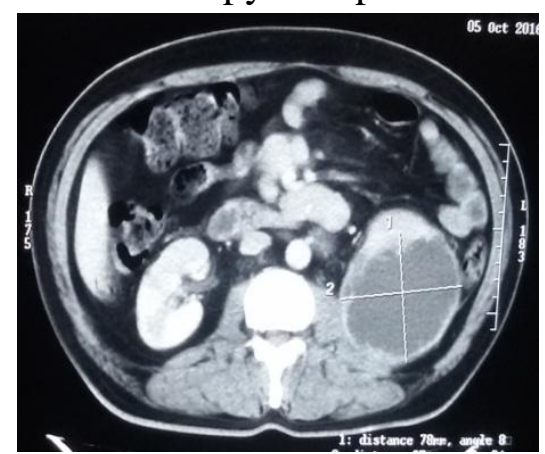

Fig - I

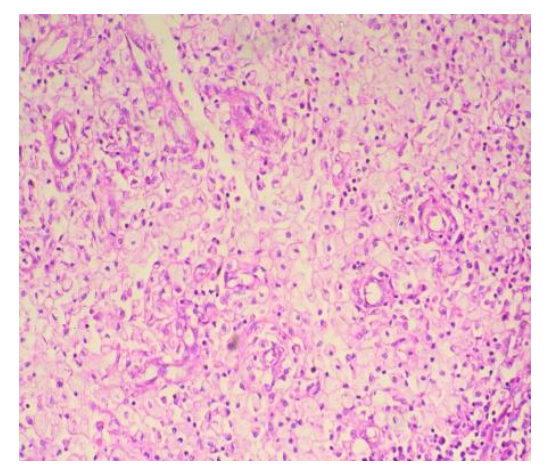

Fig - II

\section{Discussion}

Xanthogranulomatous pyelonephritis (XGP) is a chronic destructive, inflammatory lesion that is formed by accumulation of lipid-laden foamy macrophages mixed inflammatory infiltrates clefts of cholesterol in the background and fibrosis ${ }^{2}$. XGP was first described by Schlagenhaufer in 1916. XGP term was first used by Oberling. It accounts $0.6-1.4 \%$ of all renal infections with 4 times female predominance and is usually noted in the fifth and sixth decades of life. XGP is almost always unilateral; therefore azotemia and frank renal failure are uncommon ${ }^{3}$. It begins within the pelvis and calyces and subsequently extends into and destroys renal parenchyma and adjacent tissues. Fibrosis prevents the distension of the pelvicalyceal system, leading to parenchymal destruction.

The exact etiology of XGP remains uncertain. Primary factors involved in the pathogenesis of XGP are nephrolithiasis, obstruction, and infection. It has been demonstrated experimentally that primary obstruction followed by chronic infection with E. coli can lead to tissue destruction and collections of lipid material by macrophages. Other factors involved in development of XGP are venous occlusion and hemorrhage, abnormal lipid metabolism, lymphatic blockage, failure of antimicrobial therapy in UTI, altered immunologic competence, and renal ischemia ${ }^{3}$. XGP may be found without stones, obstruction and documented infection ${ }^{1}$. The gross pathological appearance of XGP is that of a mass of yellow tissue with regional necrosis and hemorrhage. Pathognomonic microscopic feature is dark sheets of lipid-laden macrophages intermixed with lymphocytes, giant cells, and plasma cells surrounding the parenchymal abscess. Cholesterol esters are thought to be derived from lyses of erythrocytes after hemorrhage. Histiocytes with foamy cytoplasm that stain positive for fat (Xanthoma cells) are hallmark of the disease ${ }^{1}$.

There are two types of XGP: diffuse XGP (80\%) in which whole of the kidney is involved and segmental XGP (20\%) in which one or two calyces or one pole of kidney is involved. XGP has been staged by Malek and Elder into 3 different stages: Stage 1, nephric, when there is only kidney involvement. Stage 2, perinephric, is when the perirenal fat is involved. The $3^{\text {rd }}$ stage, paranephric, is when there is widespread involvement of the retroperitoneal area ${ }^{4}$.

XGP patients appear chronically ill. Symptoms are nonspecific and include anorexia, flank pain (69\%), fever with chills (69\%) and persistent bacteriuria (46\%). Sixty two percent of the patients have a flank mass and $35 \%$ are associated with calculi. Other less common findings include hypertension, hematuria, or hepatomegaly ${ }^{4}$.

Laboratory parameters may reveal anemia, elevated white blood counts and elevated acute phase reactants like, erythrocyte sedimentation rate. Nonspecific hepatic dysfunction that resolves after nephrectomy is seen in $17-38 \%$ of patients. Urinalysis usually shows pus cells and protein. Urine cultures usually grow proteus and E. coli ${ }^{4}$. 
The radiological assessment of XGP is usually nonspecific but contrast enhanced computed tomography (CECT) is the most useful. CT shows in 50 to $80 \%$ classic triad of unilateral renal enlargement with little or no function and a large calculus in the renal pelvis ${ }^{[5]}$. Due to the abundant vascularity within the granulation tissue in the cystic wall, only wall enhancement is seen on contrast study and cavities do not enhance. However tumors and other inflammatory lesions usually enhance as whole, thereby helping in differentiating it from XGP. CT often shows stone in a pelvis however calyceal stones are also not uncommon and they seen to be 'suspended' within calyces due to thickness of pus in calyces ${ }^{1}$. Not infrequently a fragmented or exploded calculus is seen called 'fragmented calculus sign'. The calyces never fill with contrast even on delayed images.

The typical characteristic of focal XGP on CT is a focal bulge with one or more low attenuation collection within. A calculus may be seen in associated calyx or infundibulum, localized perinephric fat stranding may be seen along with this. XGP displays neoplasm like properties capable of local tissue invasion into spleen, pancreas, or duodenum and destruction hence were referred to as a pseudotumor. This makes XGP diagnosis difficult based on radiological assessment. Renal cell carcinoma and other solid renal lesions must be considered in the differential diagnosis. CT scan helps in surgical planning, as it can reflect the amount of extra-renal extension if any. Magnetic resonance imaging can be used to assess the extrarenal extension of inflammation ${ }^{6}$. To assess the function of kidney radionuclide renal scanning using $99 \mathrm{mTc}$-DMSA can be used and quantify the differential lack of function in the involved kidney ${ }^{7}$.

The treatment of XGP is mainly surgical. However, long-term antimicrobial therapy will eradicate the infection and restore renal function in some patients ${ }^{8}$. Nephrectomy along with resection of the involved tissues is the treatment modality of choice. Partial nepherectomy is considered in segmental and bilateral XGP. Antimicrobials should be administered prior to surgery to control local infection.

Histopathological assessment is the mainstay of diagnosis. The lipid-laden macrophages associated with XGP closely resemble clear cell adenocarcinoma and may be difficult to distinguish solely on the basis of frozen section. The inflammatory changes, as mentioned earlier along with immunohistochemistry positive to CD68 confirm the diagnosis of $\mathrm{XGP}^{2}$.

\section{Conclusion}

XGP is a serious condition that mimics different conditions including RCC. There are no specific signs on imaging and laboratory investigations diagnosis of XGP. Most features of XGP resemble radiological features of renal cell carcinoma and hence necessitate nephrectomy. Frozen section does not differentiate XGP and RCC. High index of suspicion and thorough evaluation is required to make a diagnosis of XGP preoperatively. However histopathological examination with immunohistochemistry remains the confirmatory tool. RCC is one of the main differential diagnoses of XGP which should be kept in mind.

\section{References}

1. Howard M. Pollack, Bruce L. McClennan et al. clinical Urography, second edition. 2000,952- 962.

2. Goodman M, Curry $T$, Russell $T$. Xanthogranulomatous pyelonephritis (XGP): A local disease with systemic manifestations. Report of 23 patients and review of the literature. Medicine (Baltimore) 1979;58:171-81.

3. Friedenberg MJ, Spjut HJ. Xanthogranulomatous pyelonephritis. Am J Roentgenol Radium Ther Nucl Med 1963;90:97-108.

4. Malek RS, Elder JS. Xanthogranulomatous pyelonephritis: a critical analysis of 26 cases and of the literature. J Urol. 1978 May. 119(5):589-93. [Medline] 
5. Elder J. Xanthogranulomatous pyelonephritis and gas forming infections of the urinary tract. AUA Update 1984;III.2:lesson 31.

6. Soler R, Pombo F, Gayol A, et al. Focal xanthogranulomatous pyelonephritis in a teenager: MR and CT findings. Eur J Radiol 1997;24:77-9.

7. Gregg CR, Rogers TE, Munford RS. Xanthogranulomatous pyelonephritis. Curr Clin Top Infect Dis. 1999. 19:287-304

8. Mollier S, Descotes JL, Pasquier D, et al. Pseudoneoplastic xanthogranulomatous pyelonephritis. A typical clinical presentation but unusual diagnosis and treatment. Eur Urol 1995;27:170-3. 